\title{
Risk of osteoporosis and pathologic fractures in cancer patients who underwent hematopoietic stem cell transplantation: a nationwide retrospective cohort study
}

\author{
Jiun-Nong Lin ${ }^{1,2,3}$, Hsuan-Ju Chen ${ }^{4,5}$, Chih-Hui Yang ${ }^{6}$, Chung-Hsu Lai ${ }^{3}$, Hsi-Hsun \\ Lin $^{3}$, Chao-Sung Chang ${ }^{7}$, Ji-An Liang ${ }^{8,9}$ \\ ${ }^{1}$ Department of Critical Care Medicine, E-Da Hospital, I-Shou University, Kaohsiung, Taiwan \\ ${ }^{2}$ School of Medicine, College of Medicine, I-Shou University, Kaohsiung, Taiwan \\ ${ }^{3}$ Division of Infectious Diseases, Department of Internal Medicine, E-Da Hospital, I-Shou University, Kaohsiung, Taiwan \\ ${ }^{4}$ Management Office for Health Data, China Medical University Hospital, Taichung, Taiwan \\ ${ }^{5}$ College of Medicine, China Medical University, Taichung, Taiwan \\ ${ }^{6}$ Department of Biological Science and Technology, Meiho University, Pingtung, Taiwan \\ ${ }^{7}$ Department of Hematology/Oncology, E-Da Cancer Hospital, Kaohsiung, Taiwan \\ ${ }^{8}$ Graduate Institute of Clinical Medical Science and School of Medicine, College of Medicine, China Medical University, \\ Taichung, Taiwan \\ ${ }^{9}$ Department of Radiation Oncology, China Medical University Hospital, Taichung, Taiwan
}

Correspondence to: Ji-An Liang, email: d4615@mail.cmuh.org.tw

Keywords: hematopoietic stem cell transplantation, osteoporosis, fracture, bone loss

Received: October 06, $2016 \quad$ Accepted: March 21, $2017 \quad$ Published: March 31, 2017

Copyright: Lin et al. This is an open-access article distributed under the terms of the Creative Commons Attribution License (CC-BY), which permits unrestricted use, distribution, and reproduction in any medium, provided the original author and source are credited.

\section{ABSTRACT}

Background: Long-term data on post-hematopoietic stem cell transplantation (HSCT) osteoporosis and fracture are limited. This study evaluated the long-term risk of osteoporosis and fracture in cancer patients who underwent HSCT.

Results: The incidence density rate of osteoporosis was 12.5 per 1000 personyears in the HSCT group, which was significantly higher than that in the nonHSCT group (5.65 per 1000 person-years) after adjustment for associated factors and consideration of competing risk factors (adjusted subhazard ratio, 1.48; 95\% confidence interval, 1.06-2.07). The incidence density rate of fracture was 4.89 per 1000 person-years in the HSCT group, and the risk of fracture was 1.40 times higher in the HSCT group than in the non-HSCT group (95\% confidence interval, 0.83-2.40). The vertebra was the most common site of fracture after HSCT $(68.4 \%)$. The risk of osteoporosis and fracture significantly increased in post-HSCT patients with both hematological malignancies and solid tumors. Both autologous and allogeneic HSCTs increased the risk of osteoporosis, whereas only autologous HSCT recipients had an increased risk of fracture.

Materials and Methods: This nationwide retrospective cohort study analyzed data from Taiwan's National Health Insurance Research Database. We identified an HSCT group comprising 1040 cancer patients who underwent HSCT during 2000-2008 and a non-HSCT group comprising 4160 propensity score-matched cancer patients who did not undergo HSCT. All patients were followed up until the occurrence of osteoporosis; fracture; December 31, 2011; or withdrawal from the insurance program.

Conclusions: HSCT recipients have an increased risk of osteoporosis.

\section{INTRODUCTION}

Hematopoietic stem cell transplantation (HSCT) involves the intravenous infusion of autologous or allogeneic multipotent hematopoietic stem cells for reestablishing recipients' hematopoietic and immunological functions $[1,2]$. HSCT is primarily recommended for patients with hematopoietic diseases and malignancies, 
and it has been increasingly used worldwide in the past three decades $[1,2]$.

Despite substantial progress in the HSCT technique and the subsequent care, many complications including mucositis, acute and chronic graft-versus-host disease, infections, hepatic veno-occlusive disease, lung injury, and secondary cancer are prevalent after the transplantation [2]. Bone loss is a common complication following HSCT [3-7]. The pathogenesis of osteoporosis and the subsequent fracture involves multiple factors including the transplant procedure, secondary hypogonadism, chemotherapy, radiotherapy, immunosuppressive therapies, reduced mobility and growth factors, and abnormal metabolism and absorption of calcium and vitamin D because of kidney, liver, and bowel dysfunctions $[3,8]$.

Several studies have reported bone loss after HSCT [9-15]. In contrast to data on the short-term effects of bone loss, data on the long-term effects of bone loss after HSCT are limited [7, 9, 13-15]. In Taiwan, HSCT has been a common therapy for hematopoietic diseases and malignancies since 1983 [16]. Approximately 200 transplantations have been performed annually in recent years, and more than 2200 patients have undergone HSCT until 2008 [16]. The present study analyzed the incidence and risk of osteoporosis and fracture in cancer patients who underwent HSCT by using Taiwan's National Health Insurance Research Database (NHIRD).

\section{RESULTS}

This study included 1040 cancer patients who underwent HSCT (HSCT group) and 4160 cancer patients who did not undergo HSCT (non-HSCT group; Table 1). The mean age of patients in the HSCT group was 34.8 years (standard deviation, 16.0 years); this group had male predominance $(60.8 \%)$. After propensity score matching, both the HSCT and non-HSCT groups had similar sex, age, comorbidities, and cancer types (all standardized differences $<0.1$ ).

During the follow-up period, 47 and 125 patients in the HSCT and non-HSCT groups developed osteoporosis, respectively (Table 2). The incidence density rates of osteoporosis were 12.5 and 5.65 per 1000 personyears in the HSCT and non-HSCT groups, respectively. Multivariate Cox proportional regression analysis indicated a significantly higher risk of osteoporosis in the HSCT group than in the non-HSCT group after adjustment for sex, age, comorbidity, and cancer type (adjusted hazard ratio [HR], 2.53; 95\% confidence interval [CI], 1.79-3.57). After consideration of the competing risk factor for death, the corresponding subhazard ratio (SHR) of osteoporosis was $1.48(95 \% \mathrm{CI}, 1.06-2.07)$ in the HSCT group compared with the non-HSCT group.

The sex-stratified analysis demonstrated that women had a higher incidence of osteoporosis than did men in both the HSCT and non-HSCT groups (Table 3 ).
However, women and men had a similar increased risk of osteoporosis in the HSCT and non-HSCT groups (adjusted HR, 2.59; 95\% CI, 1.64-4.09 in women; adjusted HR, $2.58 ; 95 \%$ CI, $1.51-4.41$ in men). The age-specific analysis indicated that patients who underwent HSCT had a higher risk of osteoporosis than did those who did not undergo HSCT in the age groups of $<18$ years (adjusted HR, 5.33; 95\% CI, 1.70-16.7) and 18-50 years (adjusted HR, 3.45; 95\% CI, 2.15-5.55). Irrespective of comorbidities, the HSCT group had a higher risk of osteoporosis than did the non-HSCT group (adjusted HR, 2.92 ; $95 \%$ CI, $1.84-4.63$ in patients without comorbidity; adjusted HR, 2.02; 95\% CI, 1.19-3.42 in patient with comorbidity). The risk of osteoporosis was significantly higher in patients with hematological malignancies (adjusted HR, 2.32; 95\% CI, 1.59-3.40) and solid tumors (adjusted HR, 5.92; 95\% CI, 2.34-15.0) in the HSCT group than in those in the non-HSCT group. A significant interaction was observed between HSCT and age on the occurrence of osteoporosis $(P=0.003)$. However, the interaction was not significant between HSCT and sex $(P=0.91)$, HSCT and comorbidity $(P=0.12)$, and HSCT and cancer type $(P=0.10)$. Furthermore, we examined the interaction between factors in the model developed for osteoporosis. No significant interactions were identified between age and sex $(P=0.18)$, transplant type and cancer type $(P=0.58)$, and comorbidity and age $(P=0.47)$.

A total of 19 patients in the HSCT group and 54 patients in the non-HSCT group developed fractures (Table 2). All these patients with fracture had also received a diagnosis of osteoporosis. The incidence density rates of fracture in the HSCT and non-HSCT groups were 4.89 and 2.41 per 1000 person-years, respectively. Moreover, the multivariate Cox proportional regression analysis demonstrated a significantly higher risk of fracture in the HSCT group than in the non-HSCT group (adjusted HR, 2.28; 95\% CI, 1.34-3.89). The corresponding SHR of fracture was 1.40 (95\% CI, 0.83-2.40) in the HSCT group compared with the non-HSCT group after consideration of the competing risk factor for death. The sex-stratified analysis indicated that only men who underwent HSCT had a higher risk of fracture than did those who did not undergo HSCT (adjusted HR, 3.21; 95\% CI, 1.62-6.37; Table 3). The age-specific analysis demonstrated that patients in the HSCT group had a higher risk of fracture than did those in the non-HSCT group in the age group of 18-50 years (adjusted HR, 2.68; 95\% CI, 1.26-5.72). The comorbidity-specific analysis indicated that the risk of fracture in patients without any comorbidity was higher in the HSCT group than in the non-HSCT group (adjusted HR, 2.26; 95\% CI, 1.09-4.69). Furthermore, the risk of fracture was significantly higher in patients with hematological malignancies (adjusted HR, 1.90; 95\% CI, 1.03-3.50) and solid tumors (adjusted HR, 5.79; 95\% CI, 1.67-20.1) in the HSCT group than in those in the non-HSCT group. The analysis of interaction between 
Table 1: Demographic factors and comorbidities of enrolled patients in this study

\begin{tabular}{|c|c|c|c|c|c|}
\hline & \multicolumn{2}{|c|}{ Non-HSCT group $(N=4160)$} & \multicolumn{2}{|c|}{ HSCT group $(N=1040)$} & \multirow{2}{*}{ Standardized difference } \\
\hline & $n$ & $\%$ & $n$ & $\%$ & \\
\hline Sex & & & & & $<0.001$ \\
\hline Women & 1633 & 39.3 & 408 & 39.2 & \\
\hline Men & 2527 & 60.7 & 632 & 60.8 & \\
\hline Age of HSCT (mean \pm standard deviation) (year) & \multicolumn{2}{|c|}{$36.3 \pm 20.5$} & \multicolumn{2}{|c|}{$34.8 \pm 16.0$} & 0.08 \\
\hline \multicolumn{6}{|l|}{ Comorbidity } \\
\hline Diabetes mellitus & 195 & 4.69 & 61 & 5.87 & 0.05 \\
\hline Hyperlipidemia & 478 & 11.5 & 116 & 11.2 & 0.01 \\
\hline Hypertension & 430 & 10.3 & 141 & 13.6 & 0.09 \\
\hline Coronary artery disease & 221 & 5.31 & 69 & 6.63 & 0.06 \\
\hline Depression & 159 & 3.82 & 41 & 3.94 & 0.006 \\
\hline Stroke & 50 & 1.20 & 16 & 1.54 & 0.03 \\
\hline Chronic obstructive pulmonary disease & 301 & 7.24 & 80 & 7.69 & 0.02 \\
\hline Chronic kidney disease & 288 & 6.92 & 81 & 7.79 & 0.03 \\
\hline \multicolumn{6}{|l|}{ Cancer } \\
\hline Head and neck cancer & 200 & 4.81 & 50 & 4.81 & $<0.001$ \\
\hline Digestive system cancers & 24 & 0.58 & 6 & 0.58 & $<0.001$ \\
\hline Lung and mediastinum cancers & 44 & 1.06 & 11 & 1.06 & $<0.001$ \\
\hline Bone and soft-tissue cancers & 40 & 0.96 & 10 & 0.96 & $<0.001$ \\
\hline Breast cancer & 28 & 0.67 & 7 & 0.67 & $<0.001$ \\
\hline Genitourinary tract cancers & 68 & 1.63 & 17 & 1.63 & $<0.001$ \\
\hline Hematologic malignancies & 3696 & 88.8 & 924 & 88.8 & $<0.001$ \\
\hline Others & 60 & 1.44 & 15 & 1.44 & $<0.001$ \\
\hline
\end{tabular}

HSCT: hematopoietic stem cell transplant.

Table 2: Incidence density rates and hazard ratios of osteoporosis and fracture in the HSCT group comparing with the non-HSCT group

\begin{tabular}{|c|c|c|c|c|c|c|c|c|c|c|}
\hline & \multicolumn{3}{|c|}{ Non-HSCT group } & \multicolumn{3}{|c|}{ HSCT group } & \multicolumn{2}{|c|}{ HR $(95 \% \mathrm{CI})$} & \multicolumn{2}{|c|}{ SHR $(95 \% \mathrm{CI})$} \\
\hline & Event no. & Person-years & IR & Event no. & Person-years & IR & Crude & Adjusted $^{\mathrm{a}}$ & Crude & Adjusted $^{\mathrm{a}}$ \\
\hline Osteoporosis & 125 & 22,114 & 5.65 & 47 & 3775 & 12.5 & $2.14(1.53-3.00)^{* * *}$ & $2.53(1.79-3.57)^{* * *}$ & $1.54(1.09-2.13)^{*}$ & $1.48(1.06-2.07)^{*}$ \\
\hline Fracture & 54 & 22,383 & 2.41 & 19 & 3889 & 4.89 & $1.97(1.17-3.33)^{*}$ & $2.28(1.34-3.89)^{* *}$ & $1.42(0.84-2.40)$ & $1.40(0.83-2.40)$ \\
\hline
\end{tabular}

HSCT: hematopoietic stem cell transplant; IR: incidence density rate per 1000 person-years; HR: hazard ratio; CI: confidence interval; SHR: subhazard ratio.

${ }^{a}$ Model adjusting for sex, age, diabetes mellitus, hyperlipidemia, hypertension, coronary artery disease, depression, stroke, chronic obstructive pulmonary disease, chronic kidney disease, and the type of cancer. $* P<0.05,{ }^{* *} P<0.01,{ }^{* * *} P<0.001$.

HSCT and each of the factors for fracture showed that sex $(P=0.13)$, age $(P=0.62)$, comorbidity $(P=0.79)$, and cancer type $(P=0.11)$ did not have significant interactions with HSCT. All the interactions between age and sex $(P=0.17)$, transplant type and cancer type $(P=0.52)$, and comorbidity and age $(P=0.16)$, were nonsignificant in the model developed for fracture.

The sites of fracture are listed in Table 4 . Vertebral fracture accounted for $68.4 \%$ and $55.6 \%$ of fractures in the HSCT and non-HSCT groups, respectively. The risk of vertebral fracture was significantly higher in the HSCT group than in the non-HSCT group (adjusted HR, 2.73; 95\% CI, 1.40-5.33). We analyzed the risk of osteoporosis and fracture according to the transplant types (Table 5). The risk of post-HSCT osteoporosis was high in both autologous HSCT (adjusted HR, 2.57; 95\% CI, 1.66-3.99) and allogeneic HSCT (adjusted HR, 2.41; 95\% CI, 1.51-3.87). The risk of fracture was significantly high in patients who underwent autologous HSCT (adjusted HR, 3.24; 95\% CI, 1.78-5.90); however, no significantly increased risk of fracture was observed in patients following allogeneic HSCT (adjusted HR, 1.28; 95\% CI, 0.51-3.22).

\section{DISCUSSION}

In this large-scale, nationwide, retrospective cohort study, we investigated the risk of osteoporosis and fracture in cancer patients who underwent HSCT in Taiwan. According to our literature review, no large-scale study has evaluated the risk of osteoporosis and fracture in cancer patients who underwent HSCT in the Asian population.

In our study, the HSCT group had a 2.53 times higher risk of osteoporosis and a 2.28 times higher risk of fracture than did the non-HSCT group after adjustment for sex, age, comorbidity, and cancer type. After considering the competing risk factor for death, the risk of osteoporosis was significantly higher in the HSCT group (SHR, 1.48; 95\% CI, 1.06-2.07); however, the effect of HSCT on fracture was not significant (SHR, 1.40; 95\% CI, 0.83-2.40). Several studies have reported the effect of HSCT on both osteoporosis and fracture [9-15]. For example, a large-scale study by Pundole et al. [7] reported that HSCT recipients were approximately 7-9 times more likely to develop fracture than the general population in the United States of America. The nonsignificant effect of 
Table 3: Incidence density rates and hazard ratios of osteoporosis and fracture in patients with and without HSCT stratified by sex, age, comorbidity, and underlying cancers

\begin{tabular}{|c|c|c|c|c|c|c|c|c|c|}
\hline & \multicolumn{3}{|c|}{ Non-HSCT group } & \multicolumn{3}{|c|}{ HSCT group } & \multicolumn{2}{|c|}{ HR $(95 \% \mathrm{CI})$} & \multirow{2}{*}{$P$ for interaction } \\
\hline & Event no. & Person-years & IR & Event no. & Person-years & IR & Crude & Adjusteda & \\
\hline \multicolumn{10}{|l|}{ Osteoporosis } \\
\hline Sex & & & & & & & & & 0.91 \\
\hline Women & 74 & 8915 & 8.30 & 27 & 1500 & 18.0 & $2.10(1.35-3.27)^{* *}$ & $2.59(1.64-4.09)^{* * * *}$ & \\
\hline Men & 51 & 13,199 & 3.86 & 20 & 2275 & 8.79 & $2.21(1.31-3.70)^{* *}$ & $2.58(1.51-4.41)^{* * *}$ & \\
\hline Age of HSCT (year) & & & & & & & & & 0.003 \\
\hline$<18$ & 8 & 6307 & 1.27 & 5 & 576 & 8.68 & $5.69(1.84-17.5)^{* * *}$ & $5.33(1.70-16.7)^{* *}$ & \\
\hline $18-50$ & 40 & 11162 & 3.58 & 31 & 2616 & 11.9 & $3.33(2.08-5.32)^{* * *}$ & $3.45(2.15-5.55)^{* * *}$ & \\
\hline$>50$ & 77 & 4646 & 16.6 & 11 & 582 & 18.9 & $1.10(0.59-2.06)$ & $1.10(0.58-2.10)$ & \\
\hline Comorbidity $^{\dagger}$ & & & & & & & & & 0.12 \\
\hline No & 58 & 16,854 & 3.44 & 27 & 2601 & 10.4 & $2.94(1.86-4.64)^{* * * *}$ & $2.92(1.84-4.63)^{* * * *}$ & \\
\hline Yes & 67 & 5260 & 12.7 & 20 & 1173 & 17.1 & $1.34(0.82-2.22)$ & $2.02(1.19-3.42)^{* *}$ & \\
\hline Type of cancer & & & & & & & & & 0.10 \\
\hline Hematologic malignancy & 115 & 19824 & 5.80 & 38 & 3407 & 11.2 & $1.86(1.29-2.68)^{* *}$ & $2.32(1.59-3.40)^{* * * *}$ & \\
\hline Solid tumor & 10 & 2290 & 4.37 & 9 & 368 & 24.5 & $5.85(2.36-14.5)^{* * * *}$ & $5.92(2.34-15.0)^{* * * *}$ & \\
\hline \multicolumn{10}{|l|}{ Fracture } \\
\hline Sex & & & & & & & & & 0.13 \\
\hline Women & 28 & 9107 & 3.07 & 6 & 1596 & 3.76 & $1.21(0.50-2.92)$ & $1.51(0.61-3.74)$ & \\
\hline Men & 26 & 13,276 & 1.96 & 13 & 2293 & 5.67 & $2.78(1.43-5.43)^{* *}$ & $3.21(1.62-6.37)^{* * *}$ & \\
\hline Age of HSCT (year) & & & & & & & & & 0.62 \\
\hline$<18$ & 5 & 6313 & 0.79 & 2 & 5873 & 3.41 & $3.47(0.66-18.2)$ & $3.41(0.63-18.5)$ & \\
\hline $18-50$ & 19 & 11259 & 1.69 & 11 & 2707 & 4.06 & $2.46(1.17-5.17)^{*}$ & $2.68(1.26-5.72)^{*}$ & \\
\hline$>50$ & 30 & 4811 & 6.24 & 6 & 595 & 10.1 & $1.53(0.64-3.67)$ & $1.64(0.67-4.02)$ & \\
\hline Comorbidity $^{\mathrm{b}}$ & & & & & & & & & 0.79 \\
\hline No & 28 & 16,983 & 1.65 & 10 & 2687 & 3.72 & $2.34(1.09-4.61)^{*}$ & $2.26(1.09-4.69)^{*}$ & \\
\hline Yes & 26 & 5400 & 4.81 & 9 & 1203 & 7.48 & $1.52(0.71-3.25)$ & $1.94(0.88-4.28)$ & \\
\hline Type of cancer & & & & & & & & & 0.11 \\
\hline Hematologic malignancy & 49 & 20087 & 2.44 & 14 & 3504 & 3.99 & $1.58(0.87-2.87)$ & $1.90(1.03-3.50)^{*}$ & \\
\hline Solid tumor & 5 & 2297 & 2.18 & 5 & 385 & 13.0 & $6.26(1.80-21.7)^{* *}$ & $5.79(1.67-20.1)^{* * *}$ & \\
\hline
\end{tabular}

HSCT: hematopoietic stem cell transplant; IR: incidence density rate per 1000 person-years; HR: hazard ratio; CI: confidence interval.

${ }^{a}$ Model mutually adjusting for sex, age, diabetes mellitus, hyperlipidemia, hypertension, coronary artery disease, depression, stroke, chronic obstructive pulmonary disease, chronic kidney disease, and the type of cancer.

'Including diabetes mellitus, hyperlipidemia, hypertension, coronary artery disease, depression, stroke, chronic obstructive pulmonary disease, and chronic kidney disease. $* P<0.05, * * P<0.01, * * * P<0.001$.

\section{Table 4: Incidence density rates and hazard ratios for different subtypes of pathologic fracture in the HSCT group comparing with the non-HSCT group}

\begin{tabular}{|c|c|c|c|c|c|c|}
\hline \multirow{2}{*}{ Subtypes of fracture (ICD-9-CM code) } & \multicolumn{2}{|c|}{ Non-HSCT group } & \multicolumn{2}{|c|}{ HSCT group } & \multicolumn{2}{|c|}{$\operatorname{HR}(95 \% \mathrm{CI})$} \\
\hline & Event no. & IR & Event no. & IR & Crude & $\begin{array}{l}\text { Adjusted }^{\mathrm{a}} \\
(\mathbf{9 5 \%} \text { CI) }\end{array}$ \\
\hline Pathologic fracture (733.1) & 4 & 0.18 & 0 & 0.00 & - & - \\
\hline Pathologic fracture, unspecified site (733.10) & 4 & 0.18 & 1 & 0.26 & $1.43(0.16-12.8)$ & $1.72(0.18-16.8)$ \\
\hline Pathologic fracture of humerus (733.11) & 4 & 0.18 & 2 & 0.51 & $2.83(0.52-15.5)$ & $3.42(0.60-19.7)$ \\
\hline Pathologic fracture of distal radius and ulna (733.12) & 0 & 0.00 & 0 & 0.00 & - & - \\
\hline Pathologic fracture of vertebrae (733.13) & 30 & 1.34 & 13 & 3.34 & $2.43(1.27-4.67)^{* * *}$ & $2.73(1.40-5.33)^{* *}$ \\
\hline Pathologic fracture of neck of femur (733.14) & 4 & 0.18 & 1 & 0.26 & $1.35(0.15-12.2)$ & $1.86(0.20-17.6)$ \\
\hline Pathologic fracture of other specified part of femur (733.15) & 2 & 0.09 & 0 & 0.00 & - & - \\
\hline Pathologic fracture of tibia or fibula (733.16) & 2 & 0.09 & 2 & 0.51 & $5.67(0.79-40.5)$ & $9.30(0.97-89.4)$ \\
\hline Pathologic fracture of other specified site (733.19) & 4 & 0.18 & 0 & 0.00 & - & - \\
\hline
\end{tabular}

HSCT: hematopoietic stem cell transplant; IR: incidence density rate per 1000 person-years; HR: hazard ratio; CI: confidence interval.

${ }^{a}$ Model adjusting for sex, age, diabetes mellitus, hyperlipidemia, hypertension, coronary artery disease, depression, stroke, chronic obstructive pulmonary disease, chronic kidney disease, and the type of cancer.

$* * P<0.01$.

HSCT on fracture in the present study may be due to a small number of fractures.

Age at the time of HSCT has an effect on bone loss after this procedure [26, 27]. Bone mineral density (BMD) constantly increases during childhood and adolescence [28]. Petryk et al. [26] reported that patients who underwent HSCT at age $<10$ years had a significantly lower BMD than did those who underwent HSCT at age $>18$ years. 
Table 5: Incidence density rates and hazard ratios of osteoporosis and fracture in different types of transplants

\begin{tabular}{|c|c|c|c|c|c|}
\hline Variable (ICD-9-CM procedure code) & $N$ & Event no. & Person-years & IR & Adjusted HR ${ }^{\mathrm{a}}(95 \% \mathrm{CI})$ \\
\hline \multicolumn{6}{|l|}{ Osteoporosis } \\
\hline Non-HSCT group & 4160 & 125 & 22,114 & 5.65 & 1 (Reference) \\
\hline \multicolumn{6}{|l|}{ HSCT group (41.0) } \\
\hline Bone marrow transplant, not otherwise specified (41.00) & 30 & 1 & 146 & 6.83 & $2.17(0.30-15.7)$ \\
\hline Autologous HSCT $(41.01,41.04,41.07,41.09)$ & 428 & 25 & 1732 & 14.4 & $2.57(1.66-3.99)^{* * *}$ \\
\hline Allogeneic HSCT $(41.02,41.03,41.05,41.08)$ & 570 & 21 & 1871 & 11.2 & $2.41(1.51-3.87)^{* * *}$ \\
\hline Cord blood stem cell transplant (41.06) & 12 & 0 & 26 & 0 & - \\
\hline \multicolumn{6}{|l|}{ Fracture } \\
\hline Non-HSCT group & 4160 & 54 & 22,383 & 2.41 & 1 (Reference) \\
\hline \multicolumn{6}{|l|}{ HSCT group (41.0) } \\
\hline Bone marrow transplant, not otherwise specified (41.00) & 30 & 0 & 154 & 0 & - \\
\hline Autologous HSCT $(41.01,41.04,41.07,41.09)$ & 428 & 14 & 1763 & 7.94 & $3.24(1.78-5.90)^{* * *}$ \\
\hline Allogeneic HSCT $(41.02,41.03,41.05,41.08)$ & 570 & 5 & 1946 & 2.57 & $1.28(0.51-3.22)$ \\
\hline Cord blood stem cell transplant (41.06) & 12 & 0 & 26 & 0 & - \\
\hline
\end{tabular}

HSCT: hematopoietic stem cell transplant; IR: incidence density rate per 1000 person-years; HR: hazard ratio; CI: confidence interval.

${ }^{a}$ Model adjusting for sex, age, diabetes mellitus, hyperlipidemia, hypertension, coronary artery disease, depression, stroke, chronic obstructive pulmonary disease, chronic kidney disease, and the type of cancer.

$* * * P<0.001$.

HSCT and its related therapy may affect bone acquisition and damage bone cells and the stromal microenvironment during the critical period of bone development [26]. In our study, the incidence of osteoporosis increased with age at HSCT receipt. A significant interaction was observed between HSCT and age on osteoporosis $(P=0.003)$. The risk of osteoporosis after HSCT was higher in patients who underwent HSCT at age $<18$ years (adjusted HR, 5.33) than in patients who underwent HSCT at the age of 18-50 years (adjusted HR, 3.45) and age $>50$ years (adjusted $\mathrm{HR}, 1.10)$. Our results are consistent with those reported by Petryk et al. [26].

Our study results revealed that vertebral fracture was the leading site of fracture in the HSCT group (68.4\%). Pundole et al. [7] also reported that vertebral fracture was the most common site of post-HSCT fracture (53\%). Bone loss can occur at any site of the body after HSCT. In one study, BMD decreased by $0.6 \%$ in the spine, $0.4 \%$ in the total body, $2.3 \%$ in the femoral neck, and $3.5 \%$ in the Ward triangle every year [13]. In the general population, the hip, forearm, and spine are the most common sites of osteoporotic facture, and vertebral fracture accounts for approximately $15 \%$ of all fractures $[29,30]$. The exact reason of the predominance of vertebral fracture after HSCT remains unknown. Additional studies are warranted to explore the cause of this result.

In the current study, the HSCT group had a higher risk of fracture than did the non-HSCT group irrespective of the presence of comorbidity. However, statistical significance was observed only in patients without comorbidity (adjusted HR, 2.26; 95\% CI, 1.09-4.69) and not in those with comorbidity (adjusted HR, 1.94; $95 \%$ CI, 0.88-4.28). A small case number of fractures in the subgroup may be a potential bias. Another possible interpretation for this phenomenon is the secondary effects of the comorbidity-based treatment. For example, corticosteroid treatment in chronic obstructive pulmonary disease can result in bone loss [31].

Studies comparing the difference in the incidence and severity of bone loss and fractures between autologous and allogeneic HSCT recipients have yielded inconsistent results. Several studies have reported a higher reduction in BMD in allogeneic HSCT recipients than in autologous HSCT recipients [32-34]. However, Yao et al. [35] revealed similar incidence and severity in BMD reduction in autologous and allogeneic HSCT recipients. Pundole et al. [7] described an approximately two-fold higher risk of fracture in autologous HSCT recipients than in allogeneic HSCT recipients. Consistent with their results, our study results revealed a higher risk of osteoporosis in autologous than in allogeneic HSCT recipients. Regarding fracture, only autologous HSCT recipients demonstrated an increased risk of fracture. Although the incidence of fracture increased in allogeneic HSCT recipients compared with the non-HSCT group, it was not statistically significant. The dose and duration of immunosuppressants as well as the use and intensity of radiotherapy can vary among patients with HSCT. These factors could affect the occurrence of osteoporotic fracture 
and may have caused a bias in our study results [3, 35]. Moreover, the occurrence rate of graft-versus-host disease can affect the risk of osteoporosis. However, no accurate information was available about these factors in the database used in this study. Therefore, we cannot conclude the reasons for the difference among these studies.

The major strengths of this study include the largescale nationwide design, comprehensive demographic data, and the long follow-up duration. However, the study has several limitations. First, the measurement of BMD through dual-energy X-ray absorptiometry is the gold standard for diagnosis of osteoporosis [36]. The NHIRD does not contain data on the BMD Z-scores or T-scores. Therefore, we could not confirm whether the diagnosis met BMD Z-scores or T-scores defined for osteoporosis. In the current study, the diagnoses of osteoporosis were based on the International Classification of Diseases, Ninth Revision, Clinical Modification (ICD-9-CM) codes, which were judged and determined by clinical physicians according to clinical standards. Moreover, a committee in the National Health Insurance comprising medical reimbursement specialists regularly reviews the charts and assesses the accuracy of these claims files. Inaccurate coding of diseases would result in no reimbursement, and the institutions would be subjected to fines. Therefore, the diagnoses and codes for osteoporosis used in this study should be correct and reliable. Second, information on several factors for osteoporosis and osteoporotic fractures, such as smoking, body mass index, calcium intake, diet supplement, physical activity, socioeconomic status, and some treatments, are unavailable in the NHIRD. These factors could have biased the study results. Third, some infections were significantly associated with osteoporosis and fracture. However, information on the laboratory confirmation of infections was unavailable in the NHIRD. Fourth, the HSCT population could have been followed up more frequently than the control group and hence might have a higher rate of osteoporosis diagnosis. This could attenuate the strength of the association between osteoporosis and HSCT. Finally, the statistical evidence derived from a retrospective study is generally weaker than that from randomized controlled trials.

In conclusion, our study results revealed a significant risk of osteoporosis following HSCT in cancer patients. Both osteoporosis and subsequent fracture largely affect the quality of life and increase morbidity and mortality. Thus, HSCT recipients should be considered at a high risk of osteoporosis, and regular follow-up and preventive measures against these complications are suggested for all HSCT recipients of cancer patients.

\section{MATERIALS AND METHODS}

\section{Data source}

Taiwan's National Health Insurance program, established in 1995, is a population-based mandatory health insurance program, covering approximately $99.9 \%$ of the Taiwanese population. In this study, we used datasets from the Registry for Catastrophic Illness Patient Database of the NHIRD. Under the National Health Insurance program, a catastrophic illness certificate is provided for patients with diseases such as cancer, end-stage renal disease, and organ transplantation. The Catastrophic Illness Patient Database is highly accurate because the certification is reviewed by relevant experts after scrupulous verification of medical records, imaging studies, and pathological findings. This database contains outpatient and inpatient information such as demographic data, visit dates, and ICD-9-CM codes of the diagnosis and procedures. For privacy reasons, all identifiable patient information in the NHIRD is encrypted before releasing it for research purposes.

\section{Ethics statement}

The NHIRD encrypts patient personal information to protect privacy and provides researchers with anonymous identification numbers associated with relevant claims information. Therefore, patient consent is not required to access the NHIRD. This study was approved to fulfill the condition for exemption by the Institutional Review Board of China Medical University (CMUH104-REC2115-CR1). The institutional review board also specifically waived the consent requirement.

\section{Study population and primary outcome}

We enrolled patients of all ages who had cancer and had undergone HSCT (ICD-9-CM for procedure code 41.0) between January 1, 2000, and December 31, 2008. The date of transplantation was defined as the index date. For comparison, patients with cancer who did not receive HSCT during the same period were randomly selected and matched with the study cohort at a 4:1 ratio by propensity score. The common comorbidities that could be associated with osteoporosis or fracture were included for analysis, namely diabetes mellitus (ICD-9-CM 250) [17], hyperlipidemia (ICD-9-CM 272) [18], hypertension (ICD-9-CM 401-405) [19], coronary artery disease (ICD9-CM 410-414) [19], depression (ICD-9-CM 296.2, $296.3,300.4,311$ ) [20], stroke (ICD-9-CM 430-438) [21], chronic obstructive pulmonary disease (ICD-9-CM 491-493, 496) [22], and chronic kidney disease (ICD-9CM 580-589) [23].

The propensity score matching, which is the logit (probability) for HSCT, was derived from a logistic regression model by using sex, age, year of the diagnosis of cancer, year of the index date, the aforementioned comorbidities, and cancer types (head and neck cancers [ICD-9-CM 140-149, 160, 161, 190-194], digestive system cancers [ICD-9-CM 150-157], lung and mediastinum cancers [ICD-9-CM 162-165], bone and soft-tissue cancers [ICD-9-CM 170-173], breast cancer 
[ICD-9-CM 174-175], genitourinary tract cancers [ICD9-CM 179-189], hematologic malignancies [ICD-9-CM 200-208], and others [ICD-9-CM 158, 159, 176, 195199]). The underlying cancers indicated for HSCT were classified into hematologic malignancies (ICD-9-CM 200-208) and solid tumors (ICD-9-CM 140-199). Patients with a history of osteoporosis and fracture and those with incomplete age or sex information in the database were excluded. In addition, to avoid fractures secondary to metastatic lesions, patients with bone metastases (ICD-9CM 198.5) were excluded.

The primary outcome was the occurrence of osteoporosis (ICD-9-CM 733.0) or pathologic fracture (ICD-9-CM 733.1) during the follow-up period. These two outcomes were followed up independently. All patients were followed up until the occurrence of the primary outcome; December 31, 2011; or withdrawal from the insurance program.

\section{Statistical analysis}

We compared sex, age, and comorbidity between the HSCT and non-HSCT groups by using standardized differences [24]. A standardized difference value $<0.1$ indicates a negligible difference in means between the two groups. The incidence density rates of osteoporosis and fracture, stratified by sex, age, and comorbidity, were calculated in both groups. The incidence density rate (per 1000 person-years) of osteoporosis or fracture was defined as the number of incident osteoporosis or fracture divided by the number of person-years. The number of personyears was calculated by summing the number of days from the index date to the date of endpoint. Furthermore, a multivariate Cox proportional hazards regression analysis was performed for measuring the adjusted HRs and $95 \%$ CIs of osteoporosis and fracture; these data were compared between the groups after adjustment for sex, age, comorbidity, and cancer type. We used an extended Cox proportional hazards model with the LunnMcNeil approach (a modified Cox proportional hazards model that considers competing risks) to evaluate the association between HSCT and the risk of osteoporosis/ fracture [25]. Finally, the interactions of HSCT with sex, age, and comorbidity status were further examined by adding their product terms into the full model for the evaluation of both osteoporosis and fracture. All analyses were conducted using the SAS statistical software (version 9.4 for Windows; SAS Institute, Inc., Cary, NC, USA), and two-tailed $P<0.05$ was considered significant.

\section{Authors' contributions}

All authors have contributed significantly, and all authors are in agreement with the content of the manuscript: Conception/design: J.-N.L., J.-A.L.; Provision of study materials: J.-A.L.; Collection and/or assembly of data: J.-N.L., H.-J.C., J.-A.L.; Data analysis and interpretation: J.-N.L., H.-J.C., C.-H.Y., C.-H.L., H.-H.L., C.-S.C., J.-A.L.; Manuscript writing: J.-N.L., H.-J.C., C.H.Y., C.-H.L., H.-H.L., C.-S.C., J.-A.L.; Final approval of manuscript: J.-N.L., H.-J.C., C.-H.Y., C.-H.L., H.-H.L., C.-S.C., J.-A.L.

\section{CONFLICTS OF INTEREST}

\author{
All authors report no conflicts of interest.
}

\section{FUNDING}

This study was supported in part by the Taiwan Ministry of Health and Welfare Clinical Trial and Research Center of Excellence (MOHW106TDU-B-212-113004); China Medical University Hospital, Academia Sinica Taiwan Biobank, Stroke Biosignature Project (BM10501010037); NRPB Stroke Clinical Trial Consortium (MOST 105-2325-B-039-003); Tseng-Lien Lin Foundation, Taichung, Taiwan; Taiwan Brain Disease Foundation, Taipei, Taiwan; Katsuzo and Kiyo Aoshima Memorial Funds, Japan; and Health and welfare surcharge of tobacco products, China Medical University Hospital Cancer Research Center of Excellence (MOHW105TDU-B-212-134003, Taiwan). The funders had no role in the study design, data collection and analysis, decision to publish, or preparation of the manuscript. No additional external funding was received for this study.

\section{REFERENCES}

1. Gratwohl A, Baldomero $\mathrm{H}$, Aljurf $\mathrm{M}$, Pasquini $\mathrm{MC}$, Bouzas LF, Yoshimi A, Szer J, Lipton J, Schwendener A, Gratwohl M, Frauendorfer K, Niederwieser D, Horowitz M, et al. Worldwide Network of Blood and Marrow Transplantation. Hematopoietic stem cell transplantation: a global perspective. JAMA. 2010; 303:1617-24.

2. Copelan EA. Hematopoietic stem-cell transplantation. N Engl J Med. 2006; 354:1813-26.

3. Tauchmanovà L, Colao A, Lombardi G, Rotoli B, Selleri C. Bone loss and its management in long-term survivors from allogeneic stem cell transplantation. J Clin Endocrinol Metab. 2007; 92:4536-45.

4. Yao S, McCarthy PL, Dunford LM, Roy DM, Brown K, Paplham P, Syta M, Lamonica D, Smiley S, Battiwalla M, Padmanabhan S, Hahn T. High prevalence of earlyonset osteopenia/osteoporosis after allogeneic stem cell transplantation and improvement after bisphosphonate therapy. Bone Marrow Transplant. 2008; 41:393-8.

5. Ebeling PR. Approach to the patient with transplantationrelated bone loss. J Clin Endocrinol Metab. 2009; 94:1483-90.

6. McClune BL, Polgreen LE, Burmeister LA, Blaes AH, Mulrooney DA, Burns LJ, Majhail NS. Screening, 
prevention and management of osteoporosis and bone loss in adult and pediatric hematopoietic cell transplant recipients. Bone Marrow Transplant. 2011; 46:1-9.

7. Pundole $\mathrm{XN}$, Barbo AG, Lin H, Champlin RE, Lu H. Increased incidence of fractures in recipients of hematopoietic stem-cell transplantation. J Clin Oncol. 2015; 33:1364-70.

8. Weilbaecher KN. Mechanisms of osteoporosis after hematopoietic cell transplantation. Biol Blood Marrow Transplant. 2000; 6:165-74.

9. Kerschan-Schindl K, Mitterbauer M, Füreder W, Kudlacek S, Grampp S, Bieglmayer C, Fialka-Moser V, Pietschmann P, Kalhs P. Bone metabolism in patients more than five years after bone marrow transplantation. Bone Marrow Transplant. 2004; 34:491-6.

10. Socié G, Mary JY, Esperou H, Robert DV, Aractingi S, Ribaud P, Devergie A, Toubert ME, Boudou P, Cathelinau B, Gluckman E, Vexiau P. Health and functional status of adult recipients 1 year after allogeneic haematopoietic stem cell transplantation. Br J Haematol. 2001; 113:194-201.

11. Kananen K, Volin L, Laitinen K, Alfthan H, Ruutu T, Välimäki MJ. Prevention of bone loss after allogeneic stem cell transplantation by calcium, vitamin $\mathrm{D}$, and sex hormone replacement with or without pamidronate. J Clin Endocrinol Metab. 2005; 90:3877-85.

12. Tauchmanovà $\mathrm{L}$, De Simone $\mathrm{G}$, Musella $\mathrm{T}$, Orio $\mathrm{F}$, Ricci $\mathrm{P}$, Nappi C, Lombardi G, Colao A, Rotoli B, Selleri C. Effects of various antireabsorptive treatments on bone mineral density in hypogonadal young women after allogeneic stem cell transplantation. Bone Marrow Transplant. 2006; 37:81-8.

13. Schulte CMS, Beelen DW. Bone loss following hematopoietic stem cell transplantation: a long-term followup. Blood. 2004; 103:3635-43.

14. Savani BN, Donohue T, Kozanas E, Shenoy A, Singh AK, Childs RW, Barrett AJ. Increased risk of bone loss without fracture risk in long-term survivors after allogeneic stem cell transplantation. Biol Blood Marrow Transplant. 2007; 13:517-20.

15. Syrjala KL, Langer SL, Abrams JR, Storer BE, Martin PJ. Late effects of hematopoietic cell transplantation among 10year adult survivors compared with case-matched controls. J Clin Oncol. 2005; 23:6596-606.

16. Chen PM, Hsiao LT, Chen MH, Chang PM, Liu CY, Hong YC, Tzeng $\mathrm{CH}$, Chiou TJ. Current status of hematopoietic stem cell transplantation in Taiwan. Bone Marrow Transplant. 2008; 42:S133-6.

17. Starup-Linde J, Vestergaard P. Management of endocrine disease: Diabetes and osteoporosis: cause for concern? Eur J Endocrinol. 2015; 173:R93-99.

18. Tarakida A, Iino K, Abe K, Taniguchi R, Higuchi T, Mizunuma H, Nakaji S. Hypercholesterolemia accelerates bone loss in postmenopausal women. Climacteric. 2011; 14:105-11.

19. Ghosh M, Majumdar SR. Antihypertensive medications, bone mineral density, and fractures: a review of old cardiac drugs that provides new insights into osteoporosis. Endocrine. 2014; 46:397-405.

20. Rosenblat JD, Gregory JM, Carvalho AF, McIntyre RS. Depression and Disturbed Bone Metabolism: A Narrative Review of the Epidemiological Findings and Postulated Mechanisms. Curr Mol Med. 2016; 16:165-78.

21. Lam FM, Bui M, Yang FZ, Pang MY. Chronic effects of stroke on hip bone density and tibial morphology: a longitudinal study. Osteoporos Int. 2016; 27:591-603.

22. Sarkar M, Bhardwaj R, Madabhavi I, Khatana J. Osteoporosis in chronic obstructive pulmonary disease. Clin Med Insights Circ Respir Pulm Med. 2015; 9:5-21.

23. Kazama JJ, Matsuo K, Iwasaki Y, Fukagawa M. Chronic kidney disease and bone metabolism. J Bone Miner Metab. 2015; 33:245-52.

24. Mamdani M, Sykora K, Li P, Normand SLT, Streiner DL, Austin PC, Rochon PA, Anderson GM. Reader's guide to critical appraisal of cohort studies: 2 . Assessing potential for confounding. BMJ. 2005; 330:960-2.

25. Lunn M, McNeil D. Applying Cox regression to competing risks. Biometrics. 1995; 51:524-32.

26. Petryk A, Polgreen LE, Zhang L, Hodges JS, Dengel DR, Hoffmeister PA, Steinberger J, Baker KS. Bone mineral deficits in recipients of hematopoietic cell transplantation: the impact of young age at transplant. Bone Marrow Transplant. 2014; 49:258-63.

27. Bechard LJ, Gordon C, Feldman HA, Venick R, Gura K, Guinan EC, Duggan C. Bone loss and vitamin D deficiency in children undergoing hematopoietic cell transplantation. Pediatr Blood Cancer. 2015; 62:687-92.

28. Boot AM, de Ridder MA, Pols HA, Krenning EP, de Muinck Keizer-Schrama SM. Bone mineral density in children and adolescents: relation to puberty, calcium intake, and physical activity. J Clin Endocrinol Metab. 1997; 82:57-62.

29. Ström O, Borgström F, Kanis JA, Compston J, Cooper C, McCloskey EV, Jönsson B. Osteoporosis: burden, health care provision and opportunities in the EU: a report prepared in collaboration with the International Osteoporosis Foundation (IOF) and the European Federation of Pharmaceutical Industry Associations (EFPIA). Arch Osteoporos. 2011; 6:59-155.

30. Johnell O, Kanis JA. An estimate of the worldwide prevalence and disability associated with osteoporotic fractures. Osteoporos Int. 2006; 17:1726-33.

31. Ogura-Tomomatsu H, Asano K, Tomomatsu K, Miyata J, Ohmori N, Kodama M, Ueda S, Takihara T, Tanaka K, Kamiishi N, Suzuki Y, Fukunaga K, Oguma T, et al. Predictors of osteoporosis and vertebral fractures in patients presenting with moderate-tosevere chronic obstructive lung disease. COPD. 2012; 9:332-7.

32. Melton LJ, Kyle RA, Achenbach SJ, Oberg AL, Rajkumar SV. Fracture risk with multiple myeloma: a population-based study. J Bone Miner Res. 2005; 20:487-93. 
33. Ebeling PR, Thomas DM, Erbas B, Hopper JL, Szer J, Grigg AP. Mechanisms of bone loss following allogeneic and autologous hemopoietic stem cell transplantation. J Bone Miner Res. 1999; 14:342-50.

34. Serio B, Pezzullo L, Fontana R, Annunziata S, Rosamilio R, Sessa M, Giudice V, Ferrara I, Rocco M, De Rosa G, Ricci $\mathrm{P}$, Tauchmanovà $\mathrm{L}$, Montuori $\mathrm{N}$, et al. Accelerated bone mass senescence after hematopoietic stem cell transplantation. Transl Med UniSa. 2013; 5:7-13.
35. Yao S, Smiley SL, West K, Lamonica D, Battiwalla M, McCarthy PL, Hahn T. Accelerated bone mineral density loss occurs with similar incidence and severity, but with different risk factors, after autologous versus allogeneic hematopoietic cell transplantation. Biol Blood Marrow Transplant. 2010; 16:1130-7.

36. Kanis JA, McCloskey EV, Johansson H, Oden A, Melton LJ, Khaltaev N. A reference standard for the description of osteoporosis. Bone. 2008; 42:467-75. 